gral qualities and a means of self-affirmation. Philanthropy helps him to become a psychologist and teacher in relation to the patient, suggests the right move from a difficult situation, leads from the state of depression that has befallen in trade failures and errors [2]. That is why it is crucial to build and develop the "human" in man.

But the doctor is not only a humanist, but a humanist. It is a conscious interaction with the people in the name of development itself and of the whole society of physicians. The role and value of humanity that the more skillfully it accumulates socio - cultural experience of human evolution and individual experience of personality development. And this is primarily due to the personal orientation of a physician on the spiritual values of mankind [5].

The main methods of formation of the personality of the physician are strong-willed efforts for the development of vocational interests, ability to resist evil, destructive to health and life of human phenomena.

It is obvious that the philosophical culture of the future doctors is necessary for the conscious and active formation of their personal qualities of a modern specialist with the scientific worldview, critical thinking, ethical culture.

Literature:

1. Abaev Yu. K. Philosophical foundations-thinking doctor. //Medical news. - 2009. - No. 4. - S. 5-12.

2. Bulletin of medical Internet conferences, 2013. Volume 3. No. 2.

3. Modern educational process in vocational school: theory and practice. Materials interuniversity scientific - methodical conference. Blagoveshchensk.2010.

4. Philosophy of medicine / Y. L. Shevchenko and others - M.: GEOTAR - MED, 2004.

5. Khrustalyov Yu. M. Philosophy of science and medicine: a textbook. - M.: GEOTAR - Media, 2009.

Coordinates for communication: Skobelkina Tatyana Nikolaevna, senior teacher of the Department of Humanities of the AGMA E - mail: tanskab2575@lenta.ru Postal address: FSBEI AGMA, Ministry of health of Russia, 675000, Blagoveshchensk, Gorkogo str., 95

UDC 612.616.2 DOI 10.22448/AMJ.2017.4.13-14

\title{
THE EFFECT OF AZITHROMYCIN ON SPERM MOTILITY AND QUANTITY OF SPERMATOZOA
}

\author{
Chi-Gun-chzhi U.A., Omelich E.V., Pchelina K.E., Barannikov S.V., Baldanov E.V., \\ Shikulskiy A.S., Grigoriev D.A., Tseluyko S.S.
}

Amur State Medical Academy, Russian Federation

Abstract The result of 38-day experiment study revealing the effect of azithromycin on the quantity and sperm motility of male albino rats is presented in the paper. The research was conducted on 30 male outbred rats weighing 200-400g aged 45 days, contained in standard vivarium conditions of the Amur State Medical Academy. Estimation of sperm motility and quantity of spermatozoa showed decrease quantity of spermatozoa in the ejaculates and decrease of their activity. That means a negative impact of azithromycin on these parameters

Key words: azithromycin, seminal fluid, spermatozoa.

Purpose of the research: to identify the effect of azithromycin on sperm motility and quantity of male adult rats spermatozoa.

Materials and methods The work was performed on 30 males of outbred rats, contained in the standard conditions of the Amur SMA vivarium. Weight of the animals had variety from 200 to $400 \mathrm{~g}$, all of them were aged 45 days.

The rats were divided into 3 groups: control group, which received only water and food; the first experimental group, which received therapeutic dose $(10 \mathrm{mg} / \mathrm{kg} / \mathrm{day})$ of the medicine and the second experimental group who received dose increased twice $(20 \mathrm{mg} / \mathrm{kg} /$ day). Each group was divided into subgroups $A$ and B.

The experiment consisted of 3 steps.

Step 1. The introduction of a substance (Azithromycin) by oral gavage in the first and the second experimental groups. Azithromycin was pre-diluted in $1 \mathrm{ml}$ isotonic solution and durated 3 days.

Step 2. The output of rats on the 4-th day in control A, the first A and the second A experimental groups.

Step 3. The output of rats on the 38-th day in control $B$, the first $B$ and the second $B$ experimental groups.

After withdrawal of rats from the experiment theirs ejaculate was obtained from the tail section of testicle by longitudinal dissection and freeding from the fat of the epididymis for motility and quantity examination.

Results The quantitative and qualitative parameters of the seminal fluid of the rats were decreased. The changes after three days of using azithromycin are uncertain. According to the results the quantitative analysis showed that in both experimental groups the number of sperm in $1 \mathrm{ml}$ of ejaculate is markedly decreased in comparison with the control group. Higher sperm motility was determined in IB and IIB experimental groups and in these groups was 
detected the highest level of atypically formed spermatozoa.

Conclusions: Due to the research it is possible to judge that azithromycin has a negative impact on sperm motility and quantity of spermatozoa. E-mail: barannikovsv97@gmail.com

\title{
DETERMINATION OF ENTRAPMENT EFFICIENCY OF CELASTROL NANOSTRUCTURED LIPID CARRIER BY MICROCOL- UMN CENTRIFUGATION
}

\author{
Bei Zhang1, Xue-Ying Yan*
}

\author{
School of Pharmacy, Heilongjiang University of Chinese Medicine, P.R.China
}

\begin{abstract}
*Corresponding author: Xue-Ying Yan, Address: Heping Road 24, Harbin 150040, School of Pharmacy, Heilongjiang University of Chinese Medicine, P.R.China, E-Mail: 1196774372@qq.com
\end{abstract}

Abstract: Celastrol, the traditional treatment of rheumatoid drugs, is a major component of Tripterygium wilfordii, and is now found to have strong broad-spectrum antitumor activity[1]. NLC have evolved from solid lipid nanoparticles, is a novel nano-drug delivery system that can significantly improve the bioavailability of liposoluble drugs, avoid leakage of encapsulated drugs during storage, increase the stability of the drug delivery system, and better control the release of drugs freed Effect, with a long cycle and slow release effect[2]. The encapsulation efficiency is an important evaluation index for NLC. The time required is short, the sample is small and the dilution factor is small, therefore, microcolumn centrifugation is often used for the separation and determination of entrapment efficiency of NLC. The researcher used dextran gel (Sephadex G-50) microcolumn centrifugation and high performance liquid chromatography (HPLC) method to determine the entrapment efficiency of celastrol NLC carrier,and achieved good results.

Key Words: Microcolumn centrifugation; Celastrol; NLC; Entrapment efficiency; HPLC

Objective Explore the optimal method of separation of NLC and free drug by microcolumn centrifugation, establish the method of determining the entrapment efficiency of celastrol NLC.

Materials and methods Instrument: Micro gel column(Self made), HPLC (Waters e2695), Sephadex G- 50 , Centrifuge(Beckman Co. Allegra 64R). Reagents:Distearin(Shyuanye Co.), Isopropyl myristate, Celastrol( Pufei De Biotech Co.), Pluronic F-68, Vitamin E-TPGS(Shyuanye Co.).

The treated Sephadex G-50 was loaded into a syringe to prepare the microgel column. The prepared blank NLC was added to the prepared microgel column to measure the recovery of blank NLC. The solution of Celastrol was added to the prepared microgel column to measure the adsorption capacity of the gypsum on the microgel column. The physical mixture of Tripterygium NLC and Celastrol aqueous solution was added to the prepared microgel column to determine the separation ability of the microgel column to the physical mixture. The Celastrol NLC was added to the prepared microgel column, and the entrapment efficiency of Celastrol NLC was determined.

Results and conclusions The HPLC conditions for the determination of Celastrol were $\mathrm{H} 2 \mathrm{O}: \mathrm{CH} 3 \mathrm{OH}=10$ : 90 , the detection wavelength was $426 \mathrm{~nm}$, and the standard curve equation was y $=14718 \mathrm{x}-1516.1(\mathrm{R} 2=0.9995)$.

Precise amount of $0.1 \mathrm{ml}$ celastrol NLC and $0.1 \mathrm{ml} 0.05 \mathrm{mg} / \mathrm{ml}$ celastrol solution, mixed, added to the prepared micro-column. Add $0.2 \mathrm{ml} \mathrm{PBS}$ buffer each time and then centrifuge, repeat the operation 20 times, collecting each eluent, placed in $2 \mathrm{ml}$ volumetric flask, add methanol to make the demulsification and constant volume to the scale. The content of celastrol in the eluate was determined by HPLC, and the elution curve was plotted by the results.

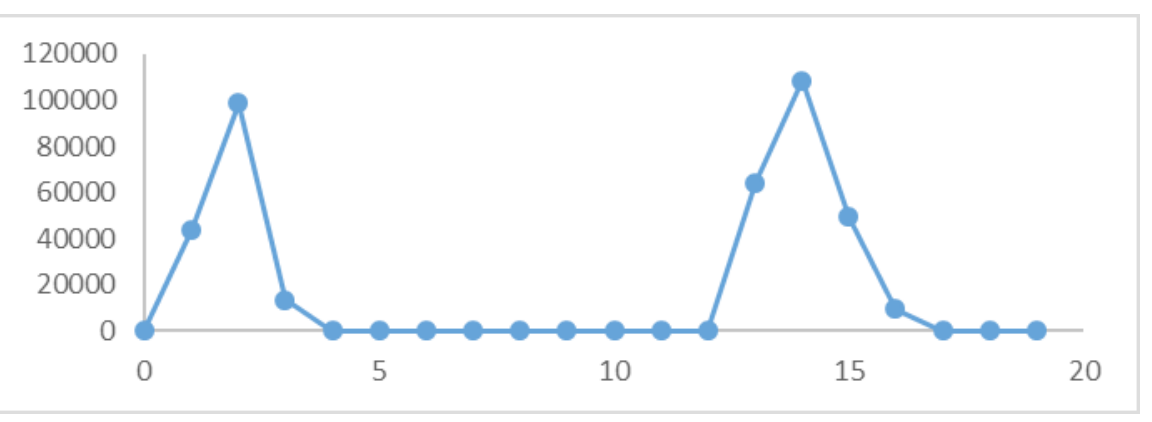

Fig. The elution curve of the physical mixture of celastrol NLC and celastrol solution, the abscissa is the elution time, and the ordinate is the peak area.

Precision extraction of $0.2 \mathrm{ml}$ celastrol NLC, adding micro-column and elution, combined with the first five times the eluent, placed in $2 \mathrm{ml}$ volumetric flask, add methanol to make the demulsification and constant volume to the scale. And then take $0.2 \mathrm{ml}$ celastrol NLC, placed in $2 \mathrm{ml}$ volumetric flask, add methanol to break the milk and set the volume to the scale. The content of celastrol was determined by HPLC, and then the entrapment efficiency was calculated. The elution was repeated three times and the entrapment efficiencies were $85.38 \%, 83.53 \%, 83.84 \%$ and $\mathrm{RSD} \%=1.18$, the entrapment efficiency is stable and favorable. 